\title{
WEAK-FOCI OF HIGH ORDER AND CYCLICITY
}

\author{
HAIHUA LIANG AND JOAN TORREGROSA
}

\begin{abstract}
A particular version of the 16th Hilbert's problem is to estimate the number, $M(n)$, of limit cycles bifurcating from a singularity of center-focus type. This paper is devoted to finding lower bounds for $M(n)$ for some concrete $n$ by studying the cyclicity of different weak-foci. Since a weak-focus with high order is the most current way to produce high cyclicity, we search for systems with the highest possible weak-focus order. For even $n$, the studied polynomial system of degree $n$ was the one obtained by [20] where the highest weak-focus order is $n^{2}+n-2$ for $n=4,6, \ldots, 18$. Moreover, we provide a system which has a weak-focus with order $(n-1)^{2}$ for $n \leq 12$. We show that Christopher's approach [5], aiming to study the cyclicity of centers, can be applied also to the weak-focus case. We also show by concrete examples that, in some families, this approach is so powerful and the cyclicity can be obtained in a simple computational way. Finally, using this approach, we obtain that $M(6) \geq 39, M(7) \geq 34$ and $M(8) \geq 63$.
\end{abstract}

\section{Introduction AND StATEMENT OF the MAIN RESUlts}

The second part of the sixteenth Hilbert's problem is concerned with the maximal number (denoted by $H(n)$ ) and relative positions of the limit cycles of planar polynomial systems of degree $n$

$$
(\dot{x}, \dot{y})=\left(P_{n}(x, y), Q_{n}(x, y)\right)
$$

This problem remains unsolved even for quadratic systems. There have been a lot of attempts to make progress in this problem, see the survey articles of Ilyashenko and $\mathrm{Li},[12,14]$. Many results have been obtained for lower bounds of $H(n)$. For example, $H(2) \geq 4, H(3) \geq 13, H(4) \geq 21, H(5) \geq 28$, and $H(6) \geq 35$. Recently, Johnson in [13] show numerically that $H(4) \geq 26$. Han and Li detail other lower bounds in [10]. In particular, developing the method used in [6] and introducing some new perturbation techniques, they improve all the existing results for $n \geq 7$ and prove that $H(n)$ grows at least as fast as $(n+2)^{2} \log (n+2) / \log 4$, see also [10].

We note that the standard technique to obtain a high number of limit cycles for a polynomial differential system is the perturbation of symmetric polynomial systems or systems with many centers or weak-foci. But there are relative few results concerning on the maximum number of limit cycles surrounding only one singularity. In the first paragraph of his paper [27], H. Zoladek said "The particular version of this (Hilbert's 16th) problem is to estimate the number $M(n)$ of small amplitude limit cycles bifurcating from an elementary center or an elementary focus. The ...p problem is ... still complicated." Clearly $M(n) \leq H(n)$.

Bautin [3] proved that $M(2)=3$. For cubic systems without quadratic terms, Sibirskii in [24] proved that no more than five limit cycles could be bifurcated from one critical point. Zoladek found an example where 11 limit cycles could be bifurcated from a single critical point of a cubic system, see [27, 28]. Christopher in [5] gave a simpler proof of Zoladek's result perturbing a Darboux cubic center. The same lower bound was also done with a different Darboux cubic center by Bondar and Sadovskii in [4]. By perturbations of a family of Darboux quartic (resp. quintic) systems inside the general quartic (resp.

2010 Mathematics Subject Classification. 34C23, 34C25, 34C07.

Key words and phrases. Weak-focus order, cyclicity, polynomial system, Lyapunov quantities. 
quintic) systems, Giné in [11] proved that $M(4) \geq 21$ (resp. $M(5) \geq 26$ ). It was also shown in [11] that $M(6) \geq 11, M(8) \geq 13$, and $M(9) \geq 16$. These result were obtained by studying polynomial differential systems whose nonlinear terms are homogeneous. Recently in [15] it is showed that $M(n) \geq n^{2}+n-2$ for $4 \leq n \leq 13$ perturbing holomorphic centers.

The cyclicity results in all the cited papers are done providing the existence of weakfoci of a given order bifurcating from a center. In all cases the limit cycles appear from a generic unfolding inside the polynomial class of a given degree. We are interested in finding explicit polynomial systems, instead of the previous existential ones, with a weakfocus of high order. In particular such systems with prescribed weak-foci should provide, if it is possible, as many limit cycles as its order.

The first result of this paper shows the systems with high cyclicity from a weak-focus of high order for $n=4,6,7$ and 8. Here system (1) is transformed to complex coordinates using the change $z=x+i y$. The systems for even degree were done in [20] and the odd degree system is new.

Theorem 1.1. (a) The cyclicity of the weak-focus of the quartic system

$$
\dot{z}=i z-2 z^{4}+z \bar{z}^{3}+i \sqrt{\frac{52278}{20723}} \bar{z}^{4}
$$

is 18 under the general quartic polynomial perturbations.

(b) There are at least 39 limit cycles bifurcating from the weak-focus of the system

$$
\dot{z}=i z-\frac{3}{2} z^{6}+z \bar{z}^{5}+i \sqrt{\frac{963010778697180}{958721342366881}} \bar{z}^{6},
$$

under general polynomial perturbations of degree 6 .

(c) There are at least 34 limit cycles bifurcating from the weak-focus of the system

$$
\dot{z}=i z+z \bar{z}^{6}+z^{7} \text {, }
$$

under general polynomial perturbations of degree 7 .

(d) There are at least 63 limit cycles bifurcating from the weak-focus of the system

$$
\dot{z}=i z-\frac{4}{3} z^{8}+z \bar{z}^{7}+i \tau_{8} \bar{z}^{8}
$$

where $\tau_{8}=\sqrt{\frac{8923642104029923643215704392751836}{14723617165367560603816000942842897}}$, under general polynomial perturbations of degree 8.

A direct consequence of the above result is the next corollary.

Corollary 1.2. For polynomial differential systems of degree $n$, we have that $M(6) \geq 39$, $M(7) \geq 34$ and $M(8) \geq 63$.

We remark that Wang and $\mathrm{Yu}$, in [26], perturb a $Z_{2}$-equivariant Hamiltonian system inside the class of polynomials of degree 6 in order to obtain $H(6) \geq 35$. Hence the limit cycles emerge from several foci. But the above result provides 39 limit cycles emerging from only one singularity.

The method to produce limit cycles from a singular point is based on the study of the Poincaré return map, see $[17,18,19]$. In this paper we assume that system (1) has an elementary monodromic singularity at the origin, i.e., the origin is an elementary center or an elementary focus. Therefore, without loss of generality, system (1) can be written using $z=x+i y$ as the complex equation

$$
\dot{z}=\left(i+\lambda_{1}\right) z+p_{n}\left(z, \bar{z}, \boldsymbol{\lambda}^{*}\right),
$$


where $\boldsymbol{\lambda}^{*}=\left(\lambda_{2}, \lambda_{3}, \ldots, \lambda_{m}\right), n, m \in \mathbb{N}^{+}$and $m \geq 2, n \geq 2$. Here $p_{n} \in \mathbb{C}\left[z, \bar{z}, \lambda_{2}, \ldots, \lambda_{m}\right]$ and $p_{n}(z, \bar{z}, \mathbf{0})$ is a polynomial of degree $n$ with only nonlinear terms. We also assume that $p_{n}\left(0,0, \boldsymbol{\lambda}^{*}\right)=\frac{\partial}{\partial z} p_{n}\left(0,0, \boldsymbol{\lambda}^{*}\right)=\frac{\partial}{\partial \bar{z}} p_{n}\left(0,0, \boldsymbol{\lambda}^{*}\right)=0$.

Clearly when $\lambda_{1} \neq 0$ equation (6) has a strong focus at the origin. When $\lambda_{1}=0$, to distinguish a center from a focus is one of the most classical problems in qualitative theory of planar ordinary differential equations, and was theoretically solved by Poincaré and Lyapunov, see $[17,18,19]$. It is the so-called center-focus problem.

The main method to deal with the center-focus problem, as well as the problem of producing limit cycles from a monodromic singularity, is the computation of Poincaré return map $\Pi: \Sigma \rightarrow \mathbb{R}$, where $\Sigma$ is a transversal section at the origin. Moreover, for analytic differential equations, the return map is analytic. Hence we can expand the return map associated to equation (6) as a Taylor series:

$$
\Pi(h)=h+\sum_{j=1}^{\infty} \alpha_{j} h^{j} .
$$

The function $d(h):=\Pi(h)-h$ is the so-called displacement function. Therefore the periodic orbits of (6) correspond to fixed points of (7) or, equivalently, to zeros of its displacement function. If all $\alpha_{j}=\alpha_{j}(\boldsymbol{\lambda})\left(\boldsymbol{\lambda}=\left(\lambda_{1}, \ldots, \lambda_{m}\right)\right)$ vanish, then we have a center at the origin. Otherwise, we have a weak-focus. Detailed calculations show that $\alpha_{1}=\mathrm{e}^{2 \pi \lambda_{1}}-1$. Moreover, when $\lambda_{1}=0$, all $\alpha_{j}(\boldsymbol{\lambda})=\alpha_{j}\left(\boldsymbol{\lambda}^{*}\right)$ are polynomials in the ring $\mathbb{C}\left[\lambda_{2}, \ldots, \lambda_{m}\right]$. Let $\beta_{1}=2 \pi \lambda_{1}$ and $\beta_{k}$ is defined from $\alpha_{k}$ modulo $\beta_{1}, \ldots, \beta_{k-1}$. It is known that since $\beta_{2 k}$ always lies in the ideal generated by the previous $\beta_{j}$ in the ring $\mathbb{C}\left[\lambda_{2}, \ldots, \lambda_{m}\right]$, the only important $\beta_{j}$ are the ones with odd subscript, see $[1$, p. 243]. We call the function $\beta_{2 j+1}$ the $j$-Lyapunov quantity and we denote it by $L(j)$.

The so-called Bautin ideal, generated by all $L(j)$ is Noetherian and hence, by the Hilbert's Basis Theorem, it is finitely generated. Hence, theoretically the center-focus problem can be solved in a finite number of steps. The main two difficulties are the number of required steps, that it is not known a priori, and the explicit calculation of the Lyapunov quantities, $L(j)$. The second one is really a hard problem to attack, even for $n=3$, because of the huge size of the Lyapunov quantities. Although, for a given family these quantities can be obtained, the solution of the system of equations necessary to characterize the centers, for example, is also very difficult to find.

If $L(k)$ is the first nonzero Lyapunov quantity, then we say that the origin is a weakfocus of order $k$. The focus order, $k$, plays an important role in the qualitative behavior of the system. In fact, according to Roussarie [21] at most $k$ limit cycles can arise from a weak-focus of order $k$, under analytic perturbations. These limit cycles are called smallamplitude limit cycles. Therefore to obtain the maximum number of them, in a concrete family, one can try to find the maximum possible focus order inside it. These families can be found solving the the polynomial systems of equations defined by the Lyapunov quantities. But the necessary number of that quantities to solve the center problem is not directly related with the maximum order of a weak-focus. In that direction, Gasull and Giné in [7] show a family for which only one Lyapunov quantity is necessary to solve the center problem but from the weak-focus can bifurcate $k$ limit cycles under analytic perturbations. This is due to the nonradicality, in that example, of the Bautin ideal. See for instance, [21] for a discussion about the cyclicity of the weak-foci in given families and the role of the radicality of the ideal formed by the Lyapunov quantities. Clearly the cyclicity of a weak-focus has a strongly dependence with the perturbed family where it is considered. Here we always perturb inside the general class of polynomials of given degree $n$. 
As we have mentioned, the maximum order is three for quadratic systems and it is five for the cubic systems without quadratic terms, see [3, 24]. For general cubic system, this maximum number would be larger than or equal to 11 , see $[4,5,27,28]$. For $n=4$ and 5 the maximal order of a weak-focus is not less than 21 and 28 respectively, see [11, 15]. For general even number $n$, the authors of [2] showed that a planar system of degree $n$ can have a weak-focus of order $n^{2}-n$. This result was improved by [16] and [20] saying that for every integer $n \geq 3$, there exists a polynomial differential system of degree $n$ having a weak-focus of order $n^{2}-1$, when $n$ is even, or $\left(n^{2}-1\right) / 2$, when $n$ is odd. The main idea to prove the above results is the computation of cyclicity of the monodromic singularity of a certain class of systems under polynomial perturbations. That is, they can not provide the concrete expression of the systems which have the mentioned focus order.

Usually it is very difficult to find a concrete system with arbitrary degree that exhibits a weak-focus of high order. For that concrete system, even with a simple form, it is also very hard to obtain the focus order for any degree. In fact, the most excellent result of [20] is a theorem which says that for every $n$ there exists a real value $\sigma_{n}$ such that equation

$$
\dot{z}=i z+z^{n}-z \bar{z}^{n-1}+i \sigma_{n} \bar{z}^{n}
$$

has a focus at the origin with focus order $n^{2}-n$ when $n$ is even and $n+1$ is a prime number or it is an integer power $p^{m}$ of a prime number $p$. The authors believe that this conclusion can be extended to general $n$ but nobody has been able to prove it.

Clearly equation (8) does not exhibit the highest weak-focus order. Qiu and Yang in [20] find other equations which seem possess weak-foci of higher order. They are respectively

$$
\dot{z}=i z-\frac{n}{n-2} z^{n}+z \bar{z}^{n-1}+i \tau_{n} \bar{z}^{n}
$$

and

$$
\dot{z}=i z+\frac{n}{n-2} z^{n}+z \bar{z}^{n-1}+\left(1+i \tilde{\tau}_{n}\right) \bar{z}^{n} .
$$

It is shown that for $n=6,8,10,12,14,16,18$ (resp. $n=7,9,11,13,15,17,19$ ) there exist values $\tau_{n}$ (resp. $\tilde{\tau}_{n}$ ) such equation (9) (resp. (10)) has a weak-focus at the origin with order $n^{2}+n-2$ (resp. $\left.\left(n^{2}+n-2\right) / 2\right)$.

To the best of our knowledge, for odd number $n$, until now the highest weak-focus order increases in the main speed of $n^{2} / 2$. One main purpose of this paper is devote to finding a system whose weak-focus order increases in the main speed of $n^{2}$. Our second main result is

Theorem 1.3. For every integer $3 \leq n \leq 12$, the origin of equation

$$
\dot{z}=i z+\bar{z}^{n-1}+z^{n}
$$

is a weak-focus of order $(n-1)^{2}$.

In the above theorem it is assumed that $n \leq 12$ simply because we have checked these cases. When we want to go further in the degree $n$, the computer need an unreasonable computing time. In fact, the calculation of the case $n=12$ needs more than 16 hours and our computer was not been able to check $n=13$ because of the memory cost ${ }^{1}$. However, we have a strong conjectural feeling that the conclusion holds for arbitrary $n$. Unfortunately we can not prove the conjecture in an analytical way.

Equation (11) differs from equations (8)-(10) and the equations in [16] in some aspects. The first is that (11) has the nonhomogeneous nonlinear terms, while all the equations investigated in both [16] and [20] have homogeneous nonlinearities. The second is that (11) has a simpler form, taking into account the number of monomials. The third is that

\footnotetext{
${ }^{1}$ The computations are done with MAPLE 18 in a Xeon computer (CPU E5-450, 3.0 GHz, RAM 32 $\mathrm{Gb})$ with GNU Linux.
} 
the order, $(n-1)^{2}$, does not depend on the parity of the degree. The inspiration for obtaining equation (11) comes from [8], where the center problem for polynomial systems with two monomial nonlinearities is considered.

The reason why we cannot check the order for high values as for equations (9) and (10) is the following. We know that if an equation has homogeneous nonlinear terms, then its weak-focus order "jump" in the step by step Lyapunov quantities computation. More precisely, if $p_{n}(z, \bar{z})$ is a homogeneous polynomial of degree $n$ and $L_{k}$ is the $k$-Lyapunov quantity of equation $\dot{z}=i z+p_{n}(z, \bar{z})$ at the origin, then $L_{k} \equiv 0$ when $2 k /(n-1)$ is not an integer, see [25]. This property make the calculation of weak-focus order easier than the nonhomogeneous cases. In fact, we have checked the weak-focus order for (9) for relative large $n(n \leq 34)$, see Proposition 3.1. But we are unable to do for (11) due to the intractable calculations.

Usually the weak-focus of high order is one of best method to produce high cyclicity examples under perturbation. Therefore equation (9) and (11) are good candidates to provide good lower bounds of $M(n)$ for even $n$ and for odd $n$ respectively. In this paper we will study the cyclicity of these two classes of equations under polynomial perturbation of degree $n$ for some concrete $n$.

The rest of the present paper is organized as follows. In Section 2 we provide a simple computational approach to deal with the cyclicity of a weak-focus. We extend the approach of Christopher, see [5], originally developed for the center cyclicity problem, to weak-foci. Section 3 is devote to the proof of Theorem 1.1 for $n=4,6$ and 8 . We also check for $n=20,22, \ldots, 34$ that equation (9) has a weak-focus of order $n^{2}+n-2$. In Section 4 we first check the validity of Theorem 1.3. Finally we study the cyclicity of the weak-focus of (11) for $n=3,4, \ldots, 8$.

\section{Christopher's Method in CALCUlAting THE CYCliCity OF WEAK-FOCUS}

In practice the computation of Lyapunov constants from the return map is not the most efficient way to proceed. Instead we will apply the method which turns out to be equivalent. According to $[17,22]$, there is an analytic positive definite function $V(z, \bar{z})$ in a neighborhood of the origin such that $X(V)=\sum_{k=0}^{\infty} V_{k} r^{2 k+2}$, where $r^{2}=z \bar{z}$ and $X$ is the vector field associated to equation (6). That is, $X(V)$ is the rate of change of $V$ along the orbits of (6). The coefficient $V_{k}$ is called the $k$-Lyapunov constant of (6) at the origin. We recall that they are polynomials in the coefficients of $p_{n}$ in (6), when $V_{0}=2 \lambda_{1}=0$.

Although the choice of $V$ is not unique, from [23] we know that $V_{k}$ is determined modulo $V_{1}, \ldots, V_{k-1}$. In fact, $V_{k}$ is equivalent to $L(k)$ modulo the previous $L(j)$.

Since the ideal, $I$, generated by the Lyapunov constants $V_{j}$ has a finite number of generators, we can let $m(n)$ be the minimum number of such constants that generate $I$, and let $\tilde{V}_{1}, \tilde{V}_{2}, \ldots, \tilde{V}_{m(n)}$ be these generators. If $\tilde{V}_{0}, \tilde{V}_{1}, \tilde{V}_{2}, \ldots, \tilde{V}_{m(n)}$ have alternate signs and $0<\left|\tilde{V}_{0}\right| \ll\left|\tilde{V}_{1}\right| \ll\left|\tilde{V}_{2}\right| \ll \cdots \ll\left|\tilde{V}_{m(n)}\right|$, then equation (6) has at least $m(n)$ limit cycles bifurcating from the origin, see $[22]$.

Usually it is very difficult to find the number $m(n)$ and the corresponding generators $\tilde{V}_{0}, \tilde{V}_{1}, \ldots, \tilde{V}_{m(n)}$. But if we can choose Lyapunov constants $V_{0}, V_{p_{1}}, V_{p_{2}}, \ldots, V_{p_{k}}$ (where $\left.V_{0}=2 \lambda_{1}\right)$ independent with respect to the parameters, then we can produce $k$ limit cycles because by suitable choice of the parameters we can have that $0<\left|V_{0}\right| \ll\left|V_{p_{1}}\right| \ll\left|V_{p_{2}}\right| \ll$ $\cdots \ll\left|V_{p_{k}}\right|$ and that $V_{0}, V_{p_{1}}, V_{p_{2}}, \ldots, V_{p_{k}}$ have alternate signs. Hence, in a small enough neighborhood of the origin, the return map has $k$ simple zeros and thus (6) has $k$ limit cycles.

However, for general polynomial systems, the computation of the Lyapunov constants from $X(V)=\sum_{k=0}^{\infty} V_{k} r^{2 k+2}$ is still very complicated. Recently, Christopher has considered 
a simple computational approach to estimate the cyclicity of centers, see the following result.

Theorem $2.1([5])$. Suppose that $\mathbf{s} \in K$ (where $K$ denote the corresponding parameter space of a family of polynomial systems) is a point on the center variety and that the first $k$ of the $L(j)$ 's have independent linear parts (with respect to the expansion of $L(j)$ about $\mathbf{s})$, then $\mathbf{s}$ lies on a component of the center variety of codimension at least $k$ and there are bifurcations which produce $k-1$ limit cycles locally from the center corresponding to the parameter value $\mathbf{s}$.

The above result provides a powerful approach to calculate the cyclicity of a center. Indeed, Christopher in [5] considers the cubic center $C_{31}$ in Zoladek's classification [27]. A direct computation shows that the linear parts of $L(1), L(2), \ldots, L(11)\left(L(0)=2 \pi \lambda_{1}\right.$ already) are independent in the parameters and therefore 11 limit cycles can bifurcate from this center. This confirm the result of [27]. Applying Christopher's approach, Giné in [11] found a quartic system with 21 limit cycles bifurcating from a center. Recently, in [10], a quintic system with 28 limit cycles surrounding only one singularity is given.

If one check carefully the proof of Theorem 2.1, it is easy to see that the same conclusion holds if we replace the center with a weak-focus.

Proposition 2.2. Suppose that at $\lambda \in K$, equation (6) has a weak-focus with focus order $k$. If $L\left(k_{1}\right), L\left(k_{2}\right), \ldots, L\left(k_{\ell-1}\right)\left(0<k_{1}<\cdots<k_{\ell-1}<k\right)$ have independent linear parts (with respect to the expansion of $L(j)$ about $\lambda$ ), then there are bifurcations which produce $\ell$ limit cycles locally from the weak-focus corresponding to the parameter value $\lambda$.

Remark 2.3. Under the hypothesis of Proposition 2.2, we have actually $\ell+1$ linear independent Lyapunov quantities which begin at $L(0)=2 \pi \lambda_{1}$ and end at $L(k)$ with $L(k) \neq$ 0 at the parameter value $\lambda$. Therefore we can also get from [22] that there are bifurcations which produce $\ell$ limit cycles.

In the next two sections we will apply Proposition 2.2 to study the cyclicity of weakfoci. It is worth emphasizing that we have successfully unfolded some concrete systems such that the number of limit cycles is the same that the corresponding weak-focus order. All is done, in general, using the linear terms of Lyapunov quantities with respect to the parameters. Therefore our work further confirm the value of the Christopher's approach. We also study some other systems which are need to be considered the quadratic terms in the parameters. The reader is referred to Theorem 3.1 of [5] for more details of high order perturbations.

\section{Cyclicity of the Weak-Focus of EQUation (9)}

As we have commented before, when we perturb in the analytic class, the order and the cyclicity of a weak-focus coincide, see [21]. But this can not be true if we restrict our study to some class, for example if the polynomial perturbations have the same degree that the vector field with a weak-focus of a prescribed order. Consequently for looking high cyclicity monodromic points the main tool is to find a weak-focus with high order. Until now, in a polynomial system of degree $n$ for even number $n \geq 4$, the highest known weak-focus order is $n^{2}+n-2$. In this section we will study the number of limit cycles bifurcating from three polynomial systems of the form (9) with degrees $n=4,6,8$, under general polynomial perturbation of the same degree. We will prove that the cyclicity and the weak-focus order coincide for the quartic family. Moreover, when we perturb (9) for $n=6$ and 8 we provide lower bounds for the cyclicity of the weak-focus for these degrees. In particular we will obtain $M(6) \geq 39$ and $M(8) \geq 63$. This will be the first part of the proof of Theorem 1.1. In the next section we will finish it. 
Proof of Theorem 1.1 for even degree cases. The proof is based on Proposition 2.2. Let $V_{m}^{\ell}$ be the linear part of the $m$-Lyapunov constant $V_{m}$ with respect to the perturbation parameters. In [20], it was shown that the origin of equations (2), (3), and (5) has a weak-focus of order 18,40, and 70, respectively.

(a) Consider the general perturbation of equation (2) in the class of the quartic polynomials without constant and linear terms:

$$
\dot{z}=i z-2 z^{4}+z \bar{z}^{3}+i \sqrt{\frac{52278}{20723}} \bar{z}^{4}+\sum_{j+k=2}^{4}\left(e_{j, k}+i f_{j, k}\right) z^{j} \bar{z}^{k} .
$$

Here $e_{j, k}$ and $f_{j, k}$ are small real parameters.

By direct computation, we obtain the expressions of $V_{1}^{\ell}, \ldots, V_{17}^{\ell}$. See the Appendix. Straightforward computations show that the Jacobian matrix of $V_{1}^{\ell}, \ldots, V_{17}^{\ell}$ with respect to the parameters has rank 17 . This yields that $V_{1}^{\ell}, \ldots, V_{17}^{\ell}$ are linearly independent. Since equation (2) has a weak-focus at the origin with order 18, it follows from Proposition 2.2 that if we adding the linear perturbation $\lambda_{1} z$ to (12) then 18 limit cycles can be obtained. Therefore, the cyclicity of the weak-focus of equation (2) is 18, under general quartic polynomial perturbations.

(b) Consider the general perturbations of equation (3) in the class of polynomials of degree 6

$$
\dot{z}=i z-\frac{3}{2} z^{6}+z \bar{z}^{5}+\sqrt{\frac{963010778697180}{958721342366881}} i \bar{z}^{6}+\sum_{j+k=2}^{6}\left(e_{j, k}+f_{j, k} i\right) z^{j} \bar{z}^{k},
$$

where $e_{j, k}, f_{j, k}$ are small real parameters.

Straightforward computations show that:

(i) Equation (3) has a weak-focus at the origin with focus order 40.

(ii) The Jacobian matrix of $\left\{V_{1}^{\ell}, V_{2}^{\ell}, \ldots, V_{39}^{\ell}\right\} \backslash\left\{V_{3}^{\ell}, V_{38}^{\ell},\right\}$ with respect to the parameters has rank 37.

(iii) $V_{38}^{\ell}$ is a linear combination of $V_{8}^{\ell}, V_{13}^{\ell}, V_{18}^{\ell}, V_{23}^{\ell}, V_{28}^{\ell}, V_{33}^{\ell}$.

(iv) $V_{3}^{\ell}$ vanishes identically. But when we vanish all the parameters that appear in the other $V_{m}^{\ell}$, the quadratic part of the third Lyapunov constant $V_{3}$ is a positive definite function in two new parameters, which do not appear in the other $V_{m}^{\ell}$.

Consequently, adding the linear perturbation term $\lambda_{1} z$ to equation (13), we obtain at least 39 limit cycles. In particular, we have at least 40 independent Lyapunov constants (remember that the first one is $\left.V_{0}=2 \lambda_{1}\right)$ which are all the $V_{m}(0 \leq m \leq 40)$ except $V_{38}$. Unfortunately we are unable to obtain the second order of all $V_{j}$ to check if $V_{38}$ is independent of $V_{1}, V_{2}, \ldots, V_{37}, V_{39}$ or not.

(d) Finally consider the general perturbation of equation (5) in the class of polynomials of degree 8

$$
\dot{z}=i z-\frac{4}{3} z^{8}+z \bar{z}^{7}+i \tau_{8} \bar{z}^{8}+\sum_{j+k=2}^{8}\left(e_{j, k}+f_{j, k} i\right) z^{j} \bar{z}^{k},
$$

where $\tau_{8}$ is defined under (5) and $e_{j, k}$ and $f_{j, k}$ are small real parameters.

Straightforward computations show that:

(i) Equation (5) has a weak-focus at the origin with order 70.

(ii) The Jacobian matrix of $\left\{V_{1}^{\ell}, \ldots, V_{69}^{\ell}\right\} \backslash\left\{V_{4}^{\ell}, V_{5}^{\ell}, V_{11}^{\ell}, V_{29}^{\ell}, V_{36}^{\ell}, V_{60}^{\ell}, V_{67}^{\ell}\right\}$ with respect to the parameters has rank 62 .

(iii) $V_{4}^{\ell}, V_{5}^{\ell}$, and $V_{11}^{\ell}$ vanish identically.

(iv) $V_{29}^{\ell}$ and $V_{36}^{\ell}$ are linear combinations of $V_{1}^{\ell}, V_{8}^{\ell}, V_{15}^{\ell}$, and $V_{22}^{\ell}$. 
(v) $V_{60}^{\ell}$ and $V_{67}^{\ell}$ are linear combinations of $V_{18}^{\ell}, V_{25}^{\ell}, V_{32}^{\ell}, V_{39}^{\ell}, V_{46}^{\ell}$, and $V_{53}^{\ell}$.

Therefore, in a similar way as in the previous cases, we have at least 62 independent Lyapunov constants $\left\{V_{1}, \ldots, V_{69}\right\} \backslash\left\{V_{4}, V_{5}, V_{11}, V_{29}, V_{36}, V_{60}, V_{67}\right\}$. By adding the linear perturbation $\lambda_{1} z$ to equation (14), we obtain at least 63 limit cycles which bifurcate from the origin.

At the end of this section, we extend Proposition 1 of [20] to higher degree.

Proposition 3.1. For every $n=20,22,24, \ldots, 34$, there exists a real constant $\tau_{n}$ such that equation

$$
\dot{z}=i z-\frac{n}{n-2} z^{n}+z \bar{z}^{n-1}+i \tau_{n} \bar{z}^{n}
$$

has a weak-focus at the origin of order $n^{2}+n-2$.

We have checked the above result in the same way of [20] by using the method developed in [9]. For the sake of brevity we will not provide the detailed proof in this paper nor the explicit expression of all values of the constants $\tau_{n}$ because of the huge size of them.

\section{Cyclicity of the Weak-Focus of EQuation (11)}

In this section we present high order weak-focus for polynomial systems of odd degree. This will be the natural candidate systems to obtain high cyclicity. As we have mentioned, among the existing literature, the highest known weak-focus order is $\left(n^{2}+n-2\right) / 2$ for polynomial systems of odd degree $n$. Although this conclusion has only been confirmed for $n \leq 19$. In this section we will first provide a very simple polynomial equation of degree $n$ with a weak-focus of order exactly $(n-1)^{2}$ at the origin for $n \leq 12$. This order improve the previous results for all odd $n \geq 5$. However, we are unable to prove analytically the conclusion for general $n$ nor check, with a computer, the validity for higher values of $n$ due to the computational difficulties. The proof of Theorem 1.3 is standard and hence it is omitted here.

Let us consider the cyclicity of equation (11) under general polynomial perturbation for some concrete values of degree $n$. The following result summarizes the obtained lower bounds for the cyclicity for $3 \leq n \leq 8$. In particular this prove the remaining case of Theorem 1.1.(c).

Theorem 4.1. Under general polynomial perturbations of degree $n$, we have that

(a) for $n=3,4,5$, the cyclicity of the origin as a weak-focus of equation (11) is $(n-1)^{2}$;

(b) for $n=6,7,8$, there are at least $n^{2}-3 n+6$ limit cycles which bifurcate from the origin.

Proof. Consider the general perturbation of equation (11)

$$
\dot{z}=i z+\bar{z}^{n-1}+z^{n}+\sum_{j+k=2}^{n}\left(e_{j, k}+i f_{j, k}\right) z^{j} \bar{z}^{k},
$$

where $e_{j, k}$ and $f_{j, k}$ are small real numbers.

Notice that Theorem 1.3 guaranties that only the first $(n-1)^{2}$ Lyapunov constants are necessary to be computed. We denote by $V_{m}^{\ell}$ the linear part of each $m$-Lyapunov constant $V_{m}$ with respect to all the perturbation parameters $e_{j, k}, f_{j, k}$. For every equation (16) with $n=3,4, \ldots, 8$, by direct calculation we obtain the expressions of $V_{m}^{\ell}$ for $m=$ $1, \ldots,(n-1)^{2}$. Here we only provide the expressions for the case $n=3$. The expressions for the other values of $n$ are omitted due to the huge size of them.

$$
V_{1}^{\ell}=2 e_{2,1},
$$




$$
\begin{aligned}
V_{2}^{\ell}= & \frac{10}{3} e_{1,1}-\frac{4}{3} e_{2,0}-\frac{8}{3} e_{2,1}-2 f_{1,2}, \\
V_{3}^{\ell}= & -\frac{3}{4} e_{0,3}-\frac{25}{18} e_{1,1}+\frac{5}{9} e_{2,0}-\frac{26}{9} e_{2,1}+\frac{5}{2} f_{0,3}+\frac{21}{4} f_{1,1}-\frac{3}{2} f_{2,0}, \\
V_{4}^{\ell}= & 2+4 e_{0,2}-\frac{24}{5} e_{0,3}-\frac{1391}{135} e_{1,1}-\frac{16}{3} e_{1,2}+\frac{362}{135} e_{2,0}-\frac{8294}{135} e_{2,1} \\
& +6 e_{3,0}-\frac{7}{3} f_{0,3}+\frac{94}{15} f_{1,1}+6 f_{1,2}+\frac{16}{15} f_{2,0} .
\end{aligned}
$$

Let $R(n)$ be the rank of the Jacobian matrix of $V_{1}^{\ell}, V_{2}^{\ell}, \ldots, V_{(n-1)^{2}-1}^{\ell}$ with respect to the parameters. Straightforward computations show that:

(i) For $n=3,4$, and $5 R(n)=(n-1)^{2}-1$;

(ii) For $n=6$, we have $V_{3}^{\ell}=0$ and $R(6)=23$;

(iii) For $n=7$, we have $V_{4}^{\ell}=0,8 V_{3}^{\ell}+9 V_{9}^{\ell}=0$, and $R(7)=33$;

(iv) For $n=8$, we have $V_{4}^{\ell}=V_{5}^{\ell}=V_{11}^{\ell}=0$ and $R(8)=45$.

For $n=3,4,5$, according to statement (i), we conclude that $V_{1}^{\ell}, V_{2}^{\ell}, \ldots, V_{(n-1)^{2}-1}^{\ell}$ are linearly independent. Finally, adding the linear perturbation terms, by Proposition 2.2, we obtain $(n-1)^{2}$ limit cycles bifurcating from the origin. For these degrees the cyclicity and the order of the weak-focus coincide.

For each $n=6,7,8$, using statements (ii)-(iv) and adding the linear perturbation terms, we get from Proposition 2.2 that at least 24, 34, and 46 limit cycles bifurcate from the origin. This complete the proof.

Notice that, from the above proof, to check if the cyclicity coincide or not with the order of the weak-focus for $n=6,7$, and 8 we should study higher order terms, not only the linear ones. But we are unable to do, due to the computational difficulties. As a direct corollary of Theorem 4.1 we have that $M(7) \geq 34$.

\section{FINAL REMARKS}

We would like to point out that by Theorem $1.1(n=4)$ and Theorem $4.1(n=3,4,5)$, in some cases the precise cyclicity can be obtained by computing the linear part of the Lyapunov constant with respect to the parameters. This actually suggest that in some cases Proposition 2.2 provide a simple way to compute the cyclicity of a weak-focus.

On the other hand, although the order of the weak-focus of equations (3) and (5) are 40 and 70 respectively, we have not proved the existence of the total number of the expected limit cycles. We have not been able to obtain all the second order terms, because of the size of them, necessary to complete the higher order study developed in [5].

The unperturbed equations (2), (3) and (5) are special cases of family (15). When perturbations of this equation are considered for bigger values of $n$, then two obstacles arise. The first is that the difference between the order of the weak-focus and the number of independent linear parts of Lyapunov constants increase with $n(2,7,15$ for $n=6,8,10$, respectively). The second is that the number of Lyapunov constants such that the linear part vanish also increases $(1,3,6$ for $n=6,8,10$, respectively). Similar phenomena occur with (11). For equation (4) the number of limit cycles using the linear parts method, 35, is one less than the order of the weak-focus, 36. Moreover, as we have partially done for (13), the quadratic parts of the Lyapunov constants with respect to the parameters were impossible to get for $n \geq 8$. This is the reason that we did not obtain more limit cycles for system (14). Therefore, if we want an effective proof that guaranties $n^{2}+n-2$ limit cycles emerging from a weak-focus for $n \geq 6$, equations (15) are not so good candidates because of the difficulties in the computations. Much more if we consider that in [15] 
the existence of systems with weak-foci of order $n^{2}+n-2$ are done for $4 \leq n \leq 13$. But they are not explicit, that is, nobody knows which is the concrete system that have the weak-focus with the prescribed cyclicity. And this is not the objective of the present paper.

\section{ApPENDiX}

In this appendix we list for equation (12) the expressions of the linear terms of $V_{1}, \ldots, V_{17}$ with respect to parameters $e_{k, \ell}$ and $f_{k, \ell}$, which are denoted by $V_{1}^{\ell}, \ldots, V_{17}^{\ell}$ and $\tau_{4}=$ $\sqrt{52278 / 20723}$.

$$
\begin{aligned}
& V_{1}^{\ell}=2 e_{2,1}, \quad V_{2}^{\ell}=\frac{14}{3} f_{0,2}, \quad V_{3}^{\ell}=4 f_{1,3}-2 f_{4,0}, \quad V_{4}^{\ell}=-\frac{4609874 e_{2,1}}{932535}-\frac{81092 \tau_{4} f_{1,2}}{18285}-\frac{324368 \tau_{4} f_{3,0}}{103615}, \\
& V_{5}^{\ell}=\frac{34200551 \tau_{4} e_{1,1}}{932535}+\frac{3547775 \tau_{4} e_{2,0}}{186507}+\frac{245 f_{0,2}}{27}, \quad V_{6}^{\ell}=\frac{40546 \tau_{4} e_{2,2}}{1219}+\frac{324368 \tau_{4} e_{3,1}}{20723}+\frac{52 f_{1,3}}{9}-\frac{26 f_{4,0}}{9}, \\
& V_{7}^{\ell}=\frac{14088921 e_{0,3}}{207230}-\frac{46483254000131 e_{2,1}}{96624614025}+\frac{8656571 \tau_{4} f_{0,3}}{124338}+\frac{545288820989 \tau_{4} f_{1,2}}{96624614025}-\frac{2535767113 \tau_{4} f_{3,0}}{111447075}, \\
& V_{8}^{\ell}=\frac{1015359204729749 \tau_{4} e_{1,1}}{2608864578675}+\frac{96973804522507 \tau_{4} e_{2,0}}{417418332588}-\frac{1875405161 f_{0,2}}{1777302}-\frac{1697710624 f_{1,1}}{2797605}+\frac{8225072 f_{2,0}}{32913}, \\
& V_{9}^{\ell}=\frac{28599479465821 \tau_{4} e_{2,2}}{96624614025}+\frac{3360253480232 \tau_{4} e_{3,1}}{19324922805}-\frac{1607744387 f_{1,3}}{1824525} \\
& -\frac{19578111 f_{2,2}}{41446}+\frac{81788931 f_{3,1}}{518075}+\frac{1607744387 f_{4,0}}{3649050}, \\
& V_{10}^{\ell}=\frac{685245141925897 e_{0,3}}{483123070125}+\frac{61444076 e_{1,2}}{932535}-\frac{942066463041041797396094 e_{2,1}}{401421492429324035625}-\frac{173075032 e_{3,0}}{932535} \\
& +\frac{6169040102183203 \tau_{4} f_{0,3}}{6956972209800}+\frac{4439063775431143910104 \tau_{4} f_{1,2}}{4054762549791151875}+\frac{770608652295616002473 \tau_{4} f_{3,0}}{1351587516597050625}, \\
& V_{11}^{\ell}=\frac{507174250857197 \tau_{4} e_{0,2}}{6}-\frac{148796946342637192397987 \tau_{4} e_{1,1}}{42537663815983959060121 \tau_{4} e_{2,0}} \\
& -\frac{1397520994328992436323 f_{0,2}}{154966555973295000}-\frac{291632714062484977 f_{1,1}}{34784861049000}+\frac{162088286038153951 f_{2,0}}{38263347153900}, \\
& V_{12}^{\ell}=\frac{474804688512 \tau_{4} e_{1,3}}{631533425}-\frac{4655302384721986055261 \tau_{4} e_{2,2}}{1441693351036854000}-\frac{11895877930588874551 \tau_{4} e_{3,1}}{7208466755184270} \\
& +\frac{237402344256 \tau_{4} e_{4,0}}{631533425}-\frac{8737060779898049174 f_{1,3}}{1139459970391875}-\frac{14912640866627 f_{2,2}}{2673888690} \\
& +\frac{12134261474098043 f_{3,1}}{4723870019000}+\frac{4368530389949024587 f_{4,0}}{1139459970391875}, \\
& V_{13}^{\ell}=\frac{345533284187702862895847 e_{0,3}}{79293134307026970000}+\frac{2552471567355052 e_{1,2}}{1913167357695}+\frac{37488382485963008616384730676232077 e_{2,1}}{107061123152577791214605981250} \\
& -\frac{112202051620557199 e_{3,0}}{38263347153900}-\frac{3092766729659294084201 \tau_{4} f_{0,3}}{1441693351036854000}+\frac{83878452225491 \tau_{4} f_{2,1}}{193249228050} \\
& +\frac{19184408399455505038785438440831 \tau_{4} f_{1,2}}{2359473788486562891781950000}+\frac{7663416299760124706601816341497 \tau_{4} f_{3,0}}{786491262828854297260650000}, \\
& V_{14}^{\ell}=\frac{19959434042337831541423 \tau_{4} e_{0,2}}{3243810039832921500}-\frac{316992212707932105375296591140819 \tau_{4} e_{1,1}}{2949342235608203614727437500} \\
& -\frac{136262369472520747355182694939 \tau_{4} e_{2,0}}{2}+\frac{148108906888790075455582068536137 f_{0,2}}{206851784302616013539750000} \\
& 2268724796621695088251875+\frac{148651784302616013539750000}{20} \\
& -\frac{403489082606362985255673709 f_{1,1}}{23193241784805388725000}+\frac{37310310503120795730742847 f_{2,0}}{2319324178480538872500}, \\
& V_{15}^{\ell}=\frac{8359652081281195981 \tau_{4} e_{1,3}}{2518681605585000}-\frac{15852358810086123826691676559319 \tau_{4} e_{2,2}}{120998655819823738040100000} \\
& -\frac{1243282760604240648774842812247 \tau_{4} e_{3,1}}{19359784931171798086416000}+\frac{5644114404667660339 \tau_{4} e_{4,0}}{1259340802792500}+\frac{4782731247 f_{0,4}}{828920} \\
& +\frac{554913355452497549595135591293 f_{1,3}}{972478984953487122540000}-\frac{277460282353488570561913 f_{2,2}}{8457934326082876800} \\
& +\frac{1508945588667918984494581 f_{3,1}}{88103482563363300000}-\frac{554913355452497549595135591293 f_{4,0}}{1944957969906974245080000} \\
& V_{16}^{\ell}=-\frac{61791641824281890066423237235810739 e_{0,3}}{245123110248326255979569250000}-\frac{174370085200402289267337407 e_{1,2}}{105142696091117762220000} \\
& -\frac{779949023651773162189044926210269108656246109 e_{2,1}}{138442365677670261002033456365290000000}-\frac{2516769682025670546983935277 e_{3,0}}{105142696091117762220000}
\end{aligned}
$$




$$
\begin{aligned}
& -\frac{218129637006554380383970115107773857 \tau_{4} f_{0,3}}{641776870468345106564690400000}-\frac{50758281437301093370561 \tau_{4} f_{2,1}}{20423989139688765000} \\
& -\frac{70117336500287875140349020117819123243239 \tau_{4} f_{1,2}}{105501175207118273132265051022500000}-\frac{1220858041749894501326456167022699191 \tau_{4} f_{3,0}}{3906649569582699704674384921875}, \\
V_{17}^{\ell}= & \frac{11596013654214856209762646122359219 \tau_{4} e_{0,2}}{64177687046834510656469040000}+\frac{411156196913972318454637523801212828379061703 \tau_{4} e_{1,1}}{148123649990794055477700131635590000000} \\
& +\frac{1032002622334976572780254334594858348541929 \tau_{4} e_{2,0}}{911530153789501879862770040834400000}-\frac{3273789013662838112788678191039788464693501 f_{0,2}}{278763805898806551350205333870000000} \\
& +\frac{58245090294633444709117411761708047177 f_{1,1}}{14439979585537764897705534000000}-\frac{2755646836045754530024185213828844031 f_{2,0}}{1868703240481357810291304400000} .
\end{aligned}
$$

\section{ACKNOWLEDGEMENTS}

This work was done when H. Liang was visiting the Department of Mathematics of Universitat Autònoma de Barcelona. He is very grateful for the support and hospitality.

The first author is supported by the NSF of China (No. 11201086 and No. 11401255) and the Excellent Young Teachers Training Program for colleges and universities of Guangdong Province, China (No. Yq2013107). The second author is partially supported by the MINECO/FEDER grants MTM2008-03437, MTM2013-40998-P, and UNAB134E-1604; the AGAUR grant 2014 SGR568; and the European grants FP7-PEOPLE-2012IRSES 318999 and 316338.

\section{REFERENCES}

[1] A. A. Andronov, E. A. Leontovich, I. I. Gordon, And A. G. Mă̌r. Theory of bifurcations of dynamic systems on a plane. Halsted Press [A division of John Wiley \& Sons], New York-Toronto, Ont.; Israel Program for Scientific Translations, Jerusalem-London, 1973. Translated from the Russian.

[2] J. Bai And Y. LiU, A class of planar degree $n$ (even number) polynomial systems with a fine focus of order $n^{2}-n$, Chinese Sci. Bull., 12 (1992), 1063-1065.

[3] N. BAUTIN, On the number of limit cycles appearing with variation of the coefficients from an equilibrium state of the type of a focus or a center, Mat. Sbor. 30 (1952), 181-196; Amer. Math. Soc. Transl. 100(1954), 397-413.

[4] Y. Bondar And A. SAdovskir, On a Zoladek Theorem, Differential Equations, 44 (2008), 263-265.

[5] C. Christopher, Estimating limit cycle bifurcations from centers, Differential equations with symbolic computation, in: Trends Math., 30 (2006), 23-35.

[6] C. Christopher and N. Lloyd, Polynomial systems: A lower bound for the Hilbert numbers, Proc. R. Soc. Lond. Ser. A 450 (1995), 219-224.

[7] A. Gasull and J. Giné. Cyclicity versus center problem, Qual. Theory Dyn. Syst., 9 (2010), $101-111$.

[8] A. Gasull, J. Giné, And J. Torregrosa, Center problem for systems with two monomial nonlinearities, Preprint. 2015.

[9] A. Gasull and J. Torregrosa, A new approach to the computation of the Lyapunov constants, Comput. Appl. Math., 20 (2001), 149-177.

[10] M. Han and J. LI, Lower bounds for the Hilbert number of polynomial systems, J. Differential Equations, 252 (2012), 3278-3304.

[11] J. GINÉ, Higher order limit cycle bifurcations from non-degenerate centers. Appl. Math. Comput. 218 (2012), 8853-8860.

[12] Yu. S. Ilyashenko, Centennial history of Hilbert's 16th problem, Bull. Amer. Math. Soc. (N.S.) 39 (2002), 301-354.

[13] T. Johnson, A quartic system with twenty-six limit cycles, Experiment. Math. 20 (2011), 323-328.

[14] J. LI, Hilbert's 16th problem and bifurcations of planar polynomial vector fields, Internat. J. Bifur. Chaos 13 (2003), 47-106.

[15] H. Liang And J. Torregrosa, Parallelization of the Lyapunov constants and cyclicity for centers of planar polynomial vector fields, Preprint (2015).

[16] J. Llibre AND R. Rabanal, Planar real polynomial differential systems of degree $n>3$ having a weak-focus of high order, Rocky Mountain J. Math. 42 (2012), 657-693. 
[17] A. M. Lyapunov, The general problem of the stability of motion. Taylor \& Francis, Ltd., London, 1992. Translated from Edouard Davaux's French translation (1907) of the 1892 Russian original and edited by A. T. Fuller. Reprint of Internat. J. Control 55 (1992), no. 3.

[18] H. Poincaré, Sur l'intégration des équations différentielles du premier ordre et du premier degré I. Rend. Circ. Mat. Palermo, 5 (1891), 161-191.

[19] H. Poincaré, Sur l'intégration des équations différentielles du premier ordre et du premier degré II. Rend. Circ. Mat. Palermo, 11 (1897), 193-239.

[20] Y. QIU AND J YANG, On the focus order of planar polynomial differential equations, J. Differential Equations, 246 (2009), 3361-3379.

[21] R. Roussarie, Bifurcation of planar vector fields and Hilbert's sixteenth problem, Birkhauser-Verlag. Progr. Math. 164, Basel, 1998.

[22] S. SHI, A method of constructing cycles without contact around a weak-focus, J. Differential Equations, 52 (1981), 301-312.

[23] S. SHI, On the structure of Poincaré-Lyapunov constants for the weak-focus of polynomial vector fields, J. Differential Equations, 52 (1984), 52-57.

[24] K. SiBIRSKII, On the number of limit cycles arising from a singular point of focus or center type. Dokl. Akad. Nauk SSSR 161 (1965), 304-307 (Russian). Soviet Math. Dokl,6 (1965), 428-431.

[25] D. Wang And R. MaO, A complex algorithm for computing Lyapunov values, Random and Computational Dynamics, 2 (1994), 261-277.

[26] S. WANG AND P. YU, Bifurcation of limit cycles in a quintic Hamiltonian system under a sixth-order perturbation, Chaos, Solitons Fractals, 26 (2005), 1317-1335.

[27] H. ZOLADEK, Eleven small limit cycles in a cubic vector field, Nonlinearity 8 (1995), 843-860.

[28] H. ZoladeK, The CD45 case revisited, Preprint (2015).

Department of Computer Science, Guangdong Polytechnic Normal University, Guangzhou, Guangdong 510665, PR China

E-mail address: haiihuaa@tom.com

Departament de Matemàtiques, Universitat Autònoma de Barcelona, 08193 Bellaterra, Barcelona, Catalonia, Spain

E-mail address: torre@mat.uab.cat 